\title{
Análisis cienciométrico de las publicaciones educacionales cubanas en la WoS y Scopus (2003-2012)
}

\author{
Miguel Cruz-Ramírez*, Miguel Escalona-Reyes**, Suitberto Cabrera-García***, Mayelín Caridad Martínez-Cepena**** \\ * Vicerrectoría de Investigación y Posgrado, Universidad de Holguín Oscar Lucero Moya, Cuba. Correo-e: mcruzr@facinf.uho.edu.cu \\ ** Facultad de Informática y Matemática, Universidad de Holguín Oscar Lucero Moya, Cuba. Correo-e: mescalonar@facinf.uho.edu.cu \\ *** Departamento de Estadística, Investigación Operativa y Calidad, Universidad Politécnica de Valencia. Correo-e: suicabga@eio.upv.es \\ **** Departamento de Educación Especial, Universidad de Ciencias Pedagógicas José de la Luz y Caballero, Cuba.
}

Correo-e: cepena@ucp.ho.rimed.cu

Recibido: 02-09-2013; 2a versión: 08-01-2014.Aceptado: 22-02-2014.

Cómo citar este artículo/Citation: Cruz-Ramírez, M.; Escalona-Reyes, M.; Cabrera-García, S.; Martínez-Cepena, M. C. (2014). Análisis cienciométrico de las publicaciones educacionales cubanas en la WoS y Scopus (2003-2012). Revista Española de Documentación Científica, 37(3):e058. doi: http://dx.doi.org/10.3989/redc.2014.3.1119

Resumen: Esta investigación consiste en un análisis cienciométrico de las publicaciones educacionales cubanas, referenciadas en la WoS y Scopus durante el período 2003-2012. Mediante un procedimiento de normalización, las palabras clave se clasifican con base en las categorías generales del tesauro ERIC. La matriz de datos comprende un total de 286 observaciones con 42 variables (41 categorías generales del tesauro y el año de publicación). A partir de varios indicadores se realiza una caracterización del conjunto de publicaciones. La productividad diacrónica tiene tendencia al crecimiento, con irregularidades que son explicadas de forma hipotética. El análisis estadístico multivariado facilita la clasificación de las investigaciones reportadas, así como la identificación de patrones subyacentes que sirven para caracterizar las publicaciones afines de mayor visibilidad.

Palabras clave: Cienciometría; publicaciones científicas; investigación educacional; Web of Science; Scopus; Cuba.

\section{Scientometric analysis of Cuban educational publications in WoS and Scopus (2003-2012)}

Abstract: This research consists of a statistical analysis of Cuban educational publications indexed in WoS and Scopus during the period 2003-2012. By means of a normalization procedure, keywords are classified according to the general categories of the ERIC thesaurus. The resulting data matrix comprised a total of 286 observations with 42 variables (41 general categories from the thesaurus plus the year of publication). Using various indicators, a characterization of the set of publications is produced. Productivity over time shows a tendency for growth, with irregularities that are explained hypothetically. A multivariate statistical analysis enables the classification of the reported investigations, as well as the identification of underlying patterns which are useful for characterizing similar publications of a greater visibility.

Keywords: Scientometrics; scientific publications; education research; Web of Science; Scopus; Cuba.

Copyright: (c) 2014 CSIC. Este es un artículo de acceso abierto distribuido bajo los términos de la licencia Creative Commons Attribution-Non Commercial (by-nc) Spain 3.0. 


\section{INTRODUCCIÓN}

La República de Cuba es un pequeño país latinoamericano que cuenta con una población de poco más de 11 millones de habitantes. Durante los últimos 50 años ha existido un interés constante por elevar la calidad de la educación, materializado no solo en el acceso equitativo a los estudios, sino también en la consolidación de un proceso de formación de profesionales de la educación que incluye la actividad de ciencia, tecnología e innovación. Aproximadamente desde principios de los años 90 del siglo pasado, con el surgimiento de numerosos programas de especialidad, maestría y doctorado afines a la pedagogía y a las ciencias de la educación, la formación de investigadores de estos campos también se ha acrecentado. Estos procesos han conducido a la diversificación de los objetos de investigación, con bases filosóficas en el materialismo dialéctico y psicológicas en el enfoque histórico-cultural (González y Reyes, 2010).

A tono con la tendencia mundial de dar mayor crédito a la visibilidad de los resultados científicos, el Ministerio de Educación Superior (MES) ha implementado una política de enaltecimiento de aquellas publicaciones referenciadas en bases de datos de importancia mundial. Para ello se estableció en el año 2010 una clasificación que divide las publicaciones en cuatro grandes grupos, en dependencia de las bases de datos donde aparecen indexadas. El Grupo I comprende la Web of Science (WoS) y Scopus, como corriente principal. Esta política ha tenido una repercusión favorable en los procesos de acreditación de programas de formación posgraduada, así como de instituciones universitarias.

De acuerdo con López (1996), una importante fuente documental para el análisis del desarrollo científico de un país lo constituyen las tesis doctorales. Ello no solo refleja las aportaciones científicas y tecnológicas, sino que también da cuenta de la formación de investigadores en diferentes campos. Varios indicadores pueden tomarse en consideración en este sentido, incluso socioeconómicos (Arencibia y de Moya, 2010), pero cuando el problema consiste en estudiar el grado de difusión del conocimiento y su estructura, es preciso recurrir a otros recursos. Al respecto, Torres (2012) plantea que la productividad científica no es solo una cuestión epistemológica sino también sociológica, o sea, de conformación de comunidades científicas y de pertinencia y difusión de la producción intelectual. A tono con esta afirmación, también es sugerente estudiar el desarrollo científico con base en las publicaciones $y$, consecuentemente, por medio de indicadores cienciométricos apoyados en recursos bibliométricos.

Respecto a las ciencias sociales y humanísticas, no existe pleno acuerdo sobre la eficacia de los métodos bibliométricos basados en el recuento de publicaciones en revistas científicas y en los índices de citación, como evidencias de calidad. Son varias las razones que podrían enumerarse, más allá de la consabida escasa cobertura en el monopolio de la información. Por ejemplo, la diversidad de formas de citar y referenciar, la importancia que en estos campos se concede a la difusión del conocimiento por medio de libros y monografías, la orientación idiosincrásica de muchos estudios, el uso cada vez más frecuente de métodos cualitativos, los hábitos de publicación de la comunidad científica, entre otras razones que limitan la generalización y/o contrastación de los análisis bibliométricos (Hicks, 1999; Moed, 2005; Vallejo y otros, 2005; Archambault y Larivière, 2010). En un estudio reciente, Gantman (2011) ha abordado el problema desde una postura nacional y señala que a los obstáculos antes mencionados se suma uno más cuando se trata de un país en vías de desarrollo. No obstante, Van Leeuwen (2006) destaca la importancia de aplicar análisis bibliométricos para el desarrollo de las ciencias sociales, por sus posibilidades y ventajas.

Dentro de las ciencias sociales se enmarcan las ciencias de la educación, donde los estudios cienciométricos también han demostrado su utilidad para identificar tendencias, desarrollos, entre innumerables oportunidades investigativas. De manera especial se destacan los análisis bibliométricos de contenido, donde se encuentran estudios asociados a revistas específicas del ámbito educacional (Hemphill y otros, 2012), a grupos de revistas de este campo (Chang y otros, 2009; Lee y otros, 2009), a temáticas afines a la educación (Pérez y otros, 2009; Gonçalves, 2010; Novo y Bautista, 2012) y a la producción científica educacional de determinados contextos nacionales o regionales (Anta, 2008; Brunner y Salazar, 2012; Göktaş y otros, 2012).

Algunos estudios advierten sobre las dificultades que existen para hacer inferencias acerca del desarrollo científico educacional, particularmente cuando las investigaciones de contenido utilizan indicadores bibliométricos tradicionales. Al respecto Diem y Wolter (2013) señalan que, por los hábitos de publicación, las ciencias de la educación se asemejan más a las humanidades que a las ciencias sociales en varios países, por una mayor tendencia a publicar los resultados científicos mediante libros y monografías.

Desde una mirada más regional, Brunner y Salazar subrayan que el estudio de la producción científica educacional iberoamericana, según las bases de datos y procedimientos del método estándar, produce un resultado prácticamente invisible a escala internacional (Brunner y Salazar, 2012). Mucho se ha discutido respecto a las posibles razones de este hecho, como las barreras idiomáticas, el bajo número de revistas iberoamericanas centradas en artículos de investigación afines a este campo, el alcance local o regional de las revistas, entre otros aspectos.

En el marco de un análisis cienciométrico de tesis doctorales españolas del campo de la educación 
matemática, Fernández y otros (2003) han implementado un modelo de clasificación atendiendo a la productividad (diacrónica, personal, institucional), a las citaciones (total, según idioma, según tipo de documento, antigüedad de las citas y citación personal), y a la temática. Los estudios referidos a los dos primeros aspectos son bastante frecuentes, no así en el último caso que apunta hacia los contenidos y en primera instancia a los perfiles temáticos de las revistas. Así, por ejemplo, en el portal SJR - SCImago Journal \& Country Rank (http:// www.scimagojr.com), con la ayuda de la opción de menú "Map Generator", es posible buscar conexiones entre diversos campos del saber científico por medio de redes de co-citaciones, pero en el caso de las investigaciones sociales (en general) y educacionales (en particular) ocurre un hecho singular. Cuando la clasificación se realiza atendiendo a los perfiles temáticos, se produce un sesgo relacionado con la numerosa cantidad de revistas existentes en el campo de la salud, las cuales también publican aspectos del ámbito educacional (formación del personal de la salud, educación para la salud, entre otros). El gráfico de la Figura 1 contiene un fragmento del mapa de áreas y categorías generado en el portal SJR, con ayuda del menú "Map Generator"; en él se reflejan varias conexiones de las investigaciones educacionales cubanas indexadas en Scopus, durante el bienio 2010-2011. Las saetas han sido añadidas con el objetivo de resaltar la red de co-citaciones del área temática "Education".

Como se puede observar, la conexión regresiva para el campo de la educación es: "Education" $\leftarrow$ "Public Health, Environmental and Ocupational Health" $\leftarrow$ "Medicine (miscellaneous)". Ello sugiere que, como regularidad, las publicaciones educacionales cubanas de mayor visibilidad toman como referencia la educación para la salud y la educación médica. Realmente la conexión final con el campo de la Medicina no es una singularidad. A continuación se relacionan algunas conexiones similares de otros países en el mismo bienio, lo cual también ha sido obtenido con la ayuda del servicio "Map Generator" en el portal SJR:

1. Chile, Colombia, Venezuela y Reino Unido: Educación $\leftarrow$ Psicología $\leftarrow$ Medicina.

2. España, Canadá y Estados Unidos: Educación $\leftarrow$ Psicología $\leftarrow$ Psiquiatría y Salud Mental $\leftarrow$ Medicina.

3. Francia, Italia, China y Alemania: Educación $\leftarrow$ Psicología $\leftarrow$ Neurociencias $\leftarrow$ Medicina.

4. Japón: Educación $\leftarrow$ Psicología $\leftarrow$ Neurociencias $\leftarrow$ Biología Celular $\leftarrow$ Biología Molecular $\leftarrow$ Bioquímica $\leftarrow$ Medicina.

Aunque en todos estos casos la referencia primaria llega hasta la Medicina como campo raíz, la distancia es mayor y aparece la Psicología como conexión previa. Este hecho es natural, en el sentido de que una base importante de la investigación educacional subyace en la psicología cognitiva (Agarwal, 2012).

Por sí solas, las redes de co-citaciones no son totalmente efectivas para determinar las áreas temáticas y sus conexiones. El análisis de los contenidos puede resultar más esclarecedor, mediante técnicas estadísticas y de minería de datos asociadas a los resúmenes y palabras clave de los artículos. Sin embargo, existe un problema relacionado con el amplio número de términos que se utilizan en el campo de las investigaciones educacionales, donde no pocos se encuentran en situación de desacuerdo

Figura 1. Redes de co-citaciones de las publicaciones educacionales cubanas

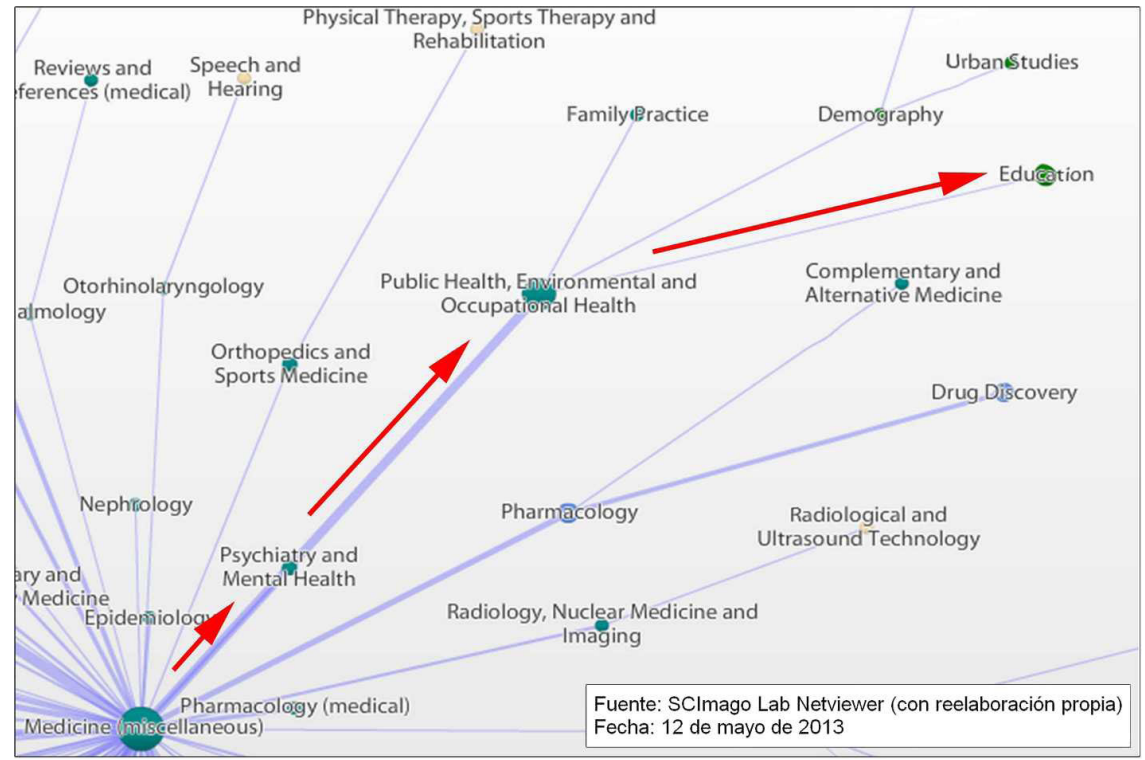


(por ejemplo, en el campo de la educación especial existe una tendencia a sustituir el término "retraso mental" por otros menos agresivos como "déficit intelectual").

En un estudio bibliométrico de la Revista Complutense de Educación, Carpallo y Burgos (2008) constatan que muchas de las palabras clave procesadas son sinónimas, pues constituyen términos sin normalizar. Ellos señalan que para eliminar el ruido o el silencio en las búsquedas documentales es conveniente utilizar listas normalizadas de términos como la Clasificación Decimal Universal, la Lista de Encabezamientos de Materias para las Bibliotecas Públicas, y los tesauros. Estos últimos, junto al título y el resumen, constituyen una primera expresión del contenido concreto de cada artículo.

Investigaciones recientes han abordado la producción científica nacional a partir de información disponible en la WoS, Scopus y en la base de datos local CubaCiencias (Araujo y otros, 2005; Arencibia y de Moya, 2010). Sin embargo, en el entramado de las investigaciones educacionales cubanas, no se tiene referencia de un análisis bibliométrico similar; mucho menos de un estudio que tome en consideración el contenido de cada publicación más allá del perfil temático de la revista. El presente estudio tiene como propósito caracterizar el contenido de las publicaciones educacionales nacionales de mayor visibilidad, enmarcadas en la década 2003-2012. El análisis se restringe a las temáticas que han sido abordadas en las investigaciones, a partir de las palabras clave de cada documento. No se desprende de aquí una disquisición sobre la producción científica cubana en materia de educación, sino de aquella que consigue mayor visibilidad por el conducto de las principales bases de datos.

Por sus especificidades, la investigación tiene un carácter exploratorio basado en el método descriptivo-explicativo. Ya que el objetivo demanda una caracterización, se presenta ésta como un conjunto de hechos esenciales y adecuadamente fundamentados. Tomando en consideración la fuente de información que se utiliza, la investigación podría catalogarse de censal-documental, por tratar de aprehender la población de publicaciones mayormente visibles en el contexto de las investigaciones educacionales locales. Sin embargo, ya que los motores de búsqueda y la metodología empleada no son infalibles, el conjunto de publicaciones estudiadas se conceptúa como muestra.

\section{MATERIALES Y MÉTODOS}

\subsection{Búsqueda en bases de datos}

Las ciencias de la educación tienen un origen relativamente reciente si se comparan con otras ciencias más consolidadas, así que la demarcación de su objeto frecuentemente es difícil de establecer. Una expresión de este fenómeno consiste en la pluralidad de formas en que muchas revistas afines pueden clasificarse dentro de las bases de datos. En el caso de la WoS, por ejemplo, la revista Journal of Educational and Behavioral Statistics aparece simultáneamente dentro de tres categorías temáticas de SSCI: Education \& Educational Research, Mathematical Psychology y Social Sciences, Mathematical Methods. Asimismo, existen varias revistas especializadas en la formación de profesionales dentro de la base de datos SCI, como International Journal of Engineering Education, la cual aparece simultáneamente dentro de las categorías Education, Scientific Disciplines y Engineering, Multidisciplinary.

Una búsqueda simultánea en la WoS y Scopus sobre documentos relacionados con la educación tiene, como primer obstáculo, el hecho de que las clasificaciones de las revistas no son totalmente equiparables. Si bien en la WoS existen categorías temáticas como las ya referidas (junto a Special Education y Educational Psychology, dentro de $\mathrm{SSCI}$ ), en Scopus pueden localizarse categorías relacionadas con la educación pero con denominaciones diferentes como Education que es muy amplia y Developmental and Educational Psychology, respectivamente dentro de las áreas Social Sciences y Psychology.

Ya que en primera instancia las ciencias de la educación se posicionan dentro del campo de las ciencias sociales, se decide realizar la búsqueda tomando como punto de partida la base de datos SSCI de la WoS y el área Social Sciences de Scopus. En el primer caso, dentro de las categorías temáticas Education \& Educational Research, Special Education y Educational Psychology, mientras que en el segundo caso la búsqueda se enmarca en la categoría Education. Las revistas referenciadas dentro de esta última categoría compensan la no inclusión de Education, Scientific Disciplines de SCI, así como otras similares en SCI-E. Esta precisión es necesaria si se concibe la formación de profesionales como parte de las ciencias de la educación, ya que "formación" es una categoría de la pedagogía.

La búsqueda se efectúa el día 9 de julio de 2013 , restringida a por lo menos un autor cubano en el área, durante el período 2003-2012 que representa una década (en el caso de la WoS este refinamiento sigue de Timespan $=2003-2012$, Countries $/$ Territories $=$ Cuba, lo cual es algo similar en Scopus). Se seleccionan las categorías Article y Conference Paper y se eliminan los registros que no poseen el resumen o las palabras clave disponibles. Con ello se producen 29 resultados en la WoS y 284 en Scopus. De forma manual, todos los datos son examinados y se eliminan siete no afines al ámbito de la investigación educacional. Después de corregir 20 repeticiones e integrar ambas fuentes de información se obtiene un total de 286 documentos. Para el conjunto de publicaciones se conforma una base de datos que contiene para cada observación el año, el título, el resumen y las palabras clave. 


\subsection{Establecimiento de la matriz de datos}

La información recopilada presenta una diversidad de palabras clave concernientes a procesos educacionales las cuales, de conjunto, aportan información sobre la naturaleza de las investigaciones reportadas. El número de palabras clave es numeroso y heterogéneo, con un promedio de 3,88 por cada publicación (1122 con 712 diferentes al eliminar repeticiones). Toda esta variedad debe su origen al uso de numerosos tesauros del campo de la educación y también al empleo de términos no normalizados.

Si se considera cada publicación como una observación muestral, con 712 variables nominales referidas a la correspondencia o no con cada una de las palabras clave identificadas, el número de variables supera notablemente el total de observaciones y ello no favorece un análisis efectivo. Por tanto, se decide reclasificar las publicaciones con base en el tesauro ERIC (http://eric.ed.gov), el cual contiene más de 11300 términos en inglés con la ventaja de aparecer agrupados en 41 áreas temáticas más generales. Existen varios tesauros para el campo de la educación (Mochón y Sorli, 2007), pero se decide seguir las categorías de ERIC por ser de referencia frecuente en las publicaciones científicas afines.

Una macro escrita en Visual Basic permite agilizar el proceso de reclasificación. Se procede de la siguiente manera:

1. Para cada publicación la macro recorre el conjunto de palabras clave. Cada una de ellas es contrastada con el total de términos de ERIC. Si el término aparece, se asigna el valor " 1 " a un campo designado para la categoría general correspondiente (41 en total), de lo contrario se asigna "0". Por ejemplo, si una palabra clave es "Student Evaluation", la macro detecta que pertenece a la categoría general "Educational Process: School Perspectives" que es la número 12. Por ello, se asigna un " 1 " al campo "C12" de la fila correspondiente. En total, de las 712 palabras clave se detectan $121(16,99 \%)$, como reflejo de una marcada falta de normalización.

2. De forma manual un dúo examina las palabras clave desechadas por la macro, por no corresponder a ningún elemento del tesauro ERIC. Para localizar una categoría afín, se recurre al resumen y en última instancia a la propia publicación. En caso de que no exista correspondencia obvia, se procede de manera inversa. O sea, se recorren las 41 categorías y se asigna un " 1 " a cada campo, según la proximidad a un área de investigación educacional correspondiente.

Siguiendo este algoritmo, a un mismo artículo le pueden corresponder ninguna, una e incluso varias categorías generales del tesauro. Finalmente resulta una matriz de datos estructurada por 286 filas que representan las publicaciones (observaciones muestrales), con 42 columnas que describen el año de aparición y la correspondencia o no con cada una de las 41 categorías del tesauro ERIC. La Tabla I contiene una descripción del conjunto de variables a investigar.

Tabla I. Descripción de las principales variables de investigación

\begin{tabular}{|c|c|c|c|}
\hline Notación & Significado & Notación & Significado \\
\hline AÑO & Año de publicación & $\mathrm{C} 21$ & El individuo en su contexto social \\
\hline $\mathrm{C} 01$ & Agricultura y fuentes naturales & $\mathrm{C} 22$ & Información y sistemas de comunicación \\
\hline $\mathrm{C} 02$ & Arte & $\mathrm{C} 23$ & Trabajo y empleo \\
\hline $\mathrm{C03}$ & Prevención y equidad & $\mathrm{C} 24$ & Comunicación y lenguaje \\
\hline $\mathrm{CO4}$ & Empresa, comercio e industria & $\mathrm{C} 25$ & Idioma \\
\hline $\mathrm{C05}$ & Medios de comunicación & $\mathrm{C} 26$ & Aprendizaje y percepción \\
\hline $\mathrm{C06}$ & Apoyo psicológico & $\mathrm{C} 27$ & Matemáticas \\
\hline $\mathrm{CO7}$ & Organización del currículo & $\mathrm{C} 28$ & Medida \\
\hline $\mathrm{C08}$ & Discapacidad & $\mathrm{C} 29$ & Salud mental \\
\hline $\mathrm{CO9}$ & Economía y finanzas & $\mathrm{C} 30$ & Ocupación \\
\hline $\mathrm{C} 10$ & Niveles educacionales, titulaciones y organizaciones & $\mathrm{C} 31$ & Personas y culturas \\
\hline $\mathrm{C} 11$ & Procesos educacionales: perspectiva del aula & $\mathrm{C} 32$ & Educación física y recreación \\
\hline $\mathrm{C} 12$ & Procesos educacionales: perspectiva de la escuela & $\mathrm{C} 33$ & Publicaciones y documentación \\
\hline C13 & Procesos educacionales: perspectiva social & C34 & Lectura \\
\hline C14 & Equipamientos & $\mathrm{C} 35$ & Investigación y teoría \\
\hline $\mathrm{C} 15$ & Instalaciones & $\mathrm{C} 36$ & Ciencia y tecnología \\
\hline $\mathrm{C} 16$ & Gobierno y política & $\mathrm{C} 37$ & Problemas sociales \\
\hline C17 & Salud y seguridad & $\mathrm{C} 38$ & Procesos y estructuras sociales \\
\hline C18 & Geografía humana & $\mathrm{C} 39$ & Estudiantes, profesores, personal docente \\
\hline C19 & Humanidades & $\mathrm{C} 40$ & Sujeto de instrucción \\
\hline $\mathrm{C} 20$ & Desarrollo y características individuales & $\mathrm{C} 41$ & Test y escalas \\
\hline
\end{tabular}


La homogenización de los datos descubre ejemplos fehacientes sobre ruidos producidos en la información, al atenerse exclusivamente al perfil temático de cada revista. Como muestra de ello, en una revista de educación médica se registran documentos afines a la superación permanente de profesores de inglés y otros relativos a la extensión universitaria, con la simple peculiaridad de tener lugar en el contexto de una universidad médica.

Es importante señalar que el procedimiento descrito no asigna palabras clave ajustadas sino una serie de categorías generales que, de conjunto, caracterizan cada documento dentro de las ciencias de la educación. Aunque se procura objetividad, no se tiene absoluta certeza sobre la validez de las palabras clave originales, pues en ocasiones los autores las seleccionan de manera inapropiada o con un inadecuado balance entre lo particular y lo general. Con el objetivo de ilustrar el procedimiento seguido, uno de los documentos analizados consta de la siguiente serie de palabras clave: "Learning; Immunochemical techniques; Pharmacy students; Poster sessions; Teaching". El procesamiento es el siguiente:

1. "Learning" es identificada por la macro dentro de la categoría "Learning and Perception". Se asigna automáticamente C26.

2. "Immunochemical techniques", aunque su empleo se adecua al perfil general de la revista, no aparece en el tesauro ERIC. Directamente se observa su conexión con "Pharmacology" y "Pharmacy", las cuales están incluidas en la categoría "Healt and Safety". Se asigna manualmente C17.

3. "Pharmacy students" no aparece en el tesauro, pero puede relacionarse con "Pharmaceutical Education" y con "Undergraduate Study". La primera está dentro de la categoría C17 que fue asignada en el caso anterior, y la segunda está contenida en la categoría "Educational Levels, Degrees, and Organizations" con lo cual se asigna manualmente C10.

4. "Poster sessions" tampoco aparece, pero ahora se trata de una terminología que no se explica por sí misma. Al consultar el resumen del artículo se obtiene más información: "...The article discusses advantages of poster sessions as teaching and learning resource, including acquiring abilities for making and presenting a poster (and hence the messages) to a scientific audience...". A partir de aquí se decide relacionar la palabra clave original con "Audiovisual Communications" y con "Student Developed Materials", ambas enmarcadas en la categoría "Communications Media". Por tanto, se asigna manualmente C05.

5. "Teaching" aparece en el tesauro como un "término muerto", en el sentido de "Occupation". La macro está programada para equiparar el término con "Teaching Methods", por ser el sentido más frecuente que se le da en el país. Por tanto, se asocia automáticamente la categoría general
"Educational Process: Clasroom Perspectives", o sea, C11.

Finalmente, el artículo se asocia con las cinco categorías generales C05, C10, C11, C17 y C26 las cuales, de conjunto, caracterizan las temáticas del campo de la educación respecto a las cuales este artículo tiene un nivel directo de relación.

\section{RESULTADOS Y DISCUSIÓN}

\subsection{Descripción general de la matriz de datos}

Aunque modesto, el total de 286 documentos es un número aceptable si se compara con otros países latinoamericanos. Por ejemplo, tomando como fuente a Scopus (Subject Area: "Social Sciences", Subject Category: "Education", Region: "All", Year: 1996-2012), SJR ubica a Cuba en el puesto 40 con 395 documentos, de ellos 390 citables. En el contexto latinoamericano ocupa el cuarto lugar, superada por Chile (617), México (708) y Brasil (3.804). Sin embargo, considerando el índice de Hirsch $(\mathrm{H}-$ index $=7$ ), Cuba pasa a ocupar el puesto 59-68 a escala mundial y el 6-7 en Latinoamérica, a causa de un menor número de citaciones. Al excluir las autocitaciones, 67 de los artículos $(17,18 \%)$ han sido citados al menos una vez y el número de citas por documento alcanza un valor promedio de 2,04, inferior a la media latinoamericana $(4,03)$ en igual período. De manera general, el número reducido de citas tiende a ser una regularidad para el campo de las investigaciones educacionales de países en vías de desarrollo, como ha sido reportado por otros autores (Vinluan, 2012). A esto se suman las fronteras naturales que impone el idioma, pues 77 $(26,92 \%)$ documentos aparecen escritos en inglés, $4(1,40 \%)$ en portugués y el resto en español.

Un total de 41 publicaciones $(14,34 \%)$ son fruto de la colaboración internacional, aspecto este que tiene tendencia a decrecer en Latinoamérica (por encima del $40 \%$ en 2003 y por debajo del $20 \%$ en 2012). También conviene destacar que el índice de coautoría alcanza el valor de 2,93 durante el transcurso de toda la década. Al examinar este indicador año por año se constata cierta estabilidad desde 2006 (cerca de tres coautores como promedio). Solo 85 artículos $(29,72 \%)$ corresponden a autores individuales y se distribuyen equitativamente. Por consiguiente, puede afirmarse que predomina el trabajo colectivo sobre el individual.

Si se toma en primera instancia el perfil de cada revista se tiene que, de los 286 documentos contabilizados, $159(55,60 \%)$ aparecen en revistas del campo de la salud (136 sobre formación inicial y permanente de profesionales de la salud y 23 sobre educación para la salud). Basándose en este criterio, muy poco se podría decir acerca de la naturaleza educacional de las investigaciones pues se hiperbolizaría el contexto donde simplemente tienen lugar. Esto reafirma la necesidad de emplear un número suficientemente grande de variables que aporten información más detallada de cada estudio reportado. 
Respecto al nivel educacional, $161(56,29 \%)$ de las publicaciones se enmarcan en la enseñanza universitaria. Este hecho corrobora la conveniencia de emplear el tesauro ERIC, pues si en su lugar se hubiese seguido, por ejemplo, el conjunto de categorías generales de EBSCO (http://www. ebscohost.com/titleLists/ehh-subject.htm), la mayoría de las publicaciones se enmarcaría dentro de la categoría "Higher Education". Con ello la reducción del número de variables sería desproporcionada y traería aparejado una pérdida excesiva de información. De forma general, la Tabla II ilustra el número de observaciones agrupadas por años, el comportamiento de las categorías estudiadas y algunos estadígrafos.

\subsection{Productividad diacrónica de publicaciones con mayor visibilidad}

La Figura 2 ilustra la producción total en ambas bases de datos durante el período examinado. El comportamiento es localmente irregular, con tendencia global al crecimiento. El ajuste exponencial (curva de regresión $y=1,80 e^{0,40 x}, R^{2}=0,69$ ) resulta discretamente mejor que el lineal. Dentro de la irregularidad pueden observarse tres crestas en 2006, 2008 y 2011. Un hecho similar ha sido reportado por Medina y otros, quienes arguyen que el patrón de crecimiento exponencial es común en áreas emergentes, donde los retrocesos ocurren "para tomar impulso renovado" (Medina y otros, 2008, p. 178).

Tabla II. Frecuencia observada de cada categoría y algunos estadígrafos

\begin{tabular}{|c|c|c|c|c|c|c|c|c|c|c|c|c|c|}
\hline \multirow{2}{*}{ Categorías } & \multicolumn{10}{|c|}{ Años } & \multicolumn{3}{|c|}{ Estadígrafos } \\
\hline & 2003 & 2004 & 2005 & 2006 & 2007 & 2008 & 2009 & 2010 & 2011 & 2012 & Suma & Media & Desv. Típ. \\
\hline $\mathrm{C} 01$ & 0 & 0 & 0 & 0 & 1 & 2 & 0 & 2 & 0 & 2 & 7 & 0.70 & 0.95 \\
\hline $\mathrm{CO2}$ & 0 & 0 & 0 & 0 & 0 & 0 & 0 & 0 & 1 & 0 & 1 & 0.10 & 0.32 \\
\hline $\mathrm{CO3}$ & 1 & 0 & 2 & 3 & 1 & 9 & 5 & 5 & 6 & 3 & 35 & 3.50 & 2.76 \\
\hline $\mathrm{CO4}$ & 0 & 0 & 0 & 0 & 1 & 3 & 2 & 0 & 0 & 1 & 7 & 0.70 & 1.06 \\
\hline $\mathrm{CO5}$ & 0 & 0 & 0 & 0 & 0 & 4 & 0 & 1 & 1 & 1 & 7 & 0.70 & 1.25 \\
\hline $\mathrm{C06}$ & 0 & 0 & 0 & 1 & 2 & 4 & 0 & 1 & 1 & 0 & 9 & 0.90 & 1.29 \\
\hline $\mathrm{C07}$ & 0 & 1 & 1 & 3 & 5 & 8 & 5 & 4 & 3 & 5 & 35 & 3.50 & 2.42 \\
\hline C08 & 1 & 0 & 0 & 1 & 0 & 0 & 0 & 0 & 0 & 3 & 5 & 0.50 & 0.97 \\
\hline C09 & 0 & 0 & 0 & 1 & 1 & 1 & 1 & 0 & 1 & 0 & 5 & 0.50 & 0.53 \\
\hline C10 & 0 & 1 & 0 & 7 & 8 & 29 & 14 & 9 & 12 & 15 & 95 & 9.50 & 8.81 \\
\hline C11 & 1 & 0 & 3 & 5 & 2 & 15 & 6 & 4 & 5 & 13 & 54 & 5.40 & 4.93 \\
\hline C12 & 0 & 0 & 0 & 1 & 0 & 3 & 2 & 2 & 1 & 3 & 12 & 1.20 & 1.23 \\
\hline C13 & 2 & 0 & 2 & 4 & 4 & 8 & 4 & 6 & 4 & 7 & 41 & 4.10 & 2.42 \\
\hline C14 & 0 & 0 & 0 & 2 & 2 & 11 & 4 & 6 & 1 & 2 & 28 & 2.80 & 3.46 \\
\hline C15 & 1 & 0 & 1 & 1 & 3 & 13 & 3 & 5 & 4 & 0 & 31 & 3.10 & 3.87 \\
\hline C16 & 1 & 0 & 2 & 2 & 2 & 15 & 6 & 6 & 7 & 3 & 44 & 4.40 & 4.40 \\
\hline C17 & 0 & 0 & 0 & 6 & 6 & 20 & 12 & 6 & 14 & 21 & 85 & 8.50 & 7.93 \\
\hline C18 & 1 & 0 & 2 & 4 & 1 & 10 & 5 & 5 & 8 & 3 & 39 & 3.90 & 3.21 \\
\hline C19 & 0 & 1 & 0 & 2 & 0 & 1 & 0 & 2 & 1 & 0 & 7 & 0.70 & 0.82 \\
\hline $\mathrm{C} 20$ & 1 & 0 & 0 & 5 & 4 & 6 & 0 & 4 & 4 & 4 & 28 & 2.80 & 2.30 \\
\hline $\mathrm{C} 21$ & 1 & 0 & 1 & 4 & 2 & 9 & 5 & 5 & 4 & 5 & 36 & 3.60 & 2.67 \\
\hline $\mathrm{C} 22$ & 0 & 0 & 0 & 2 & 0 & 6 & 0 & 3 & 3 & 2 & 16 & 1.60 & 2.01 \\
\hline C23 & 0 & 0 & 0 & 2 & 2 & 3 & 3 & 5 & 3 & 4 & 22 & 2.20 & 1.75 \\
\hline C24 & 0 & 0 & 0 & 0 & 0 & 1 & 0 & 0 & 1 & 1 & 3 & 0.30 & 0.48 \\
\hline C25 & 0 & 0 & 0 & 0 & 1 & 4 & 0 & 0 & 2 & 1 & 8 & 0.80 & 1.32 \\
\hline C26 & 0 & 0 & 3 & 6 & 3 & 12 & 4 & 3 & 12 & 10 & 53 & 5.30 & 4.55 \\
\hline C27 & 0 & 0 & 0 & 0 & 0 & 1 & 1 & 0 & 1 & 3 & 6 & 0.60 & 0.97 \\
\hline C28 & 0 & 0 & 0 & 6 & 4 & 7 & 4 & 5 & 1 & 0 & 27 & 2.70 & 2.79 \\
\hline C29 & 0 & 0 & 0 & 0 & 0 & 2 & 0 & 0 & 3 & 1 & 6 & 0.60 & 1.07 \\
\hline C30 & 0 & 0 & 0 & 4 & 5 & 7 & 5 & 5 & 5 & 3 & 34 & 3.40 & 2.55 \\
\hline C31 & 1 & 0 & 1 & 2 & 0 & 14 & 5 & 4 & 2 & 1 & 30 & 3.00 & 4.19 \\
\hline C32 & 1 & 0 & 0 & 0 & 0 & 0 & 0 & 0 & 1 & 1 & 3 & 0.30 & 0.48 \\
\hline C33 & 0 & 0 & 0 & 0 & 1 & 5 & 2 & 4 & 1 & 2 & 15 & 1.50 & 1.78 \\
\hline C34 & 0 & 0 & 0 & 0 & 0 & 1 & 0 & 1 & 0 & 0 & 2 & 0.20 & 0.42 \\
\hline C35 & 0 & 0 & 0 & 6 & 3 & 12 & 3 & 5 & 6 & 3 & 38 & 3.80 & 3.71 \\
\hline C36 & 0 & 0 & 3 & 3 & 3 & 17 & 6 & 6 & 6 & 10 & 54 & 5.40 & 5.08 \\
\hline C37 & 1 & 0 & 2 & 1 & 0 & 13 & 3 & 5 & 3 & 2 & 30 & 3.00 & 3.83 \\
\hline C38 & 1 & 0 & 2 & 6 & 1 & 11 & 6 & 10 & 6 & 3 & 46 & 4.60 & 3.84 \\
\hline C39 & 0 & 0 & 0 & 3 & 4 & 6 & 8 & 3 & 8 & 8 & 40 & 4.00 & 3.37 \\
\hline $\mathrm{C} 40$ & 1 & 0 & 0 & 4 & 3 & 8 & 2 & 3 & 13 & 6 & 40 & 4.00 & 4.06 \\
\hline C41 & 0 & 0 & 0 & 5 & 2 & 7 & 2 & 4 & 5 & 2 & 27 & 2.70 & 2.45 \\
\hline
\end{tabular}


Figura 2. Publicaciones educacionales cubanas en la WoS y Scopus (2003-2012)

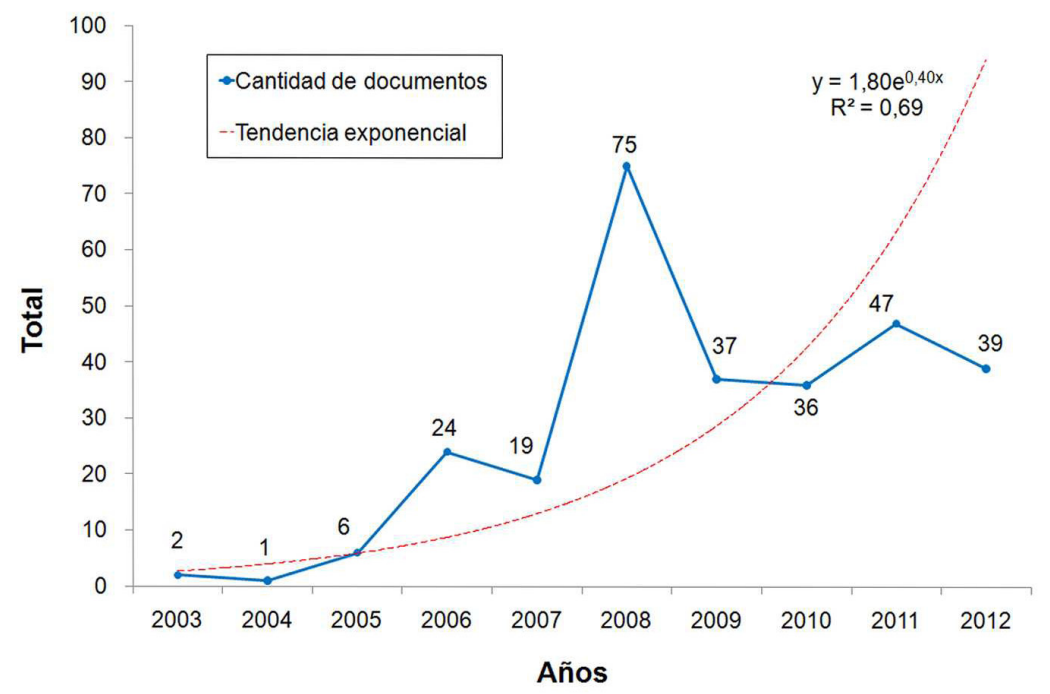

A fin de indagar acerca de las causas de esta aparente estacionalidad, en la presente investigación se examinan los declives 2006-2007, 2008-2009 y 2011-2012. De forma manual se tabulan los datos referidos a los autores, pues los reportes de sus nombres aparecen de forma heterogénea, mientras que otros son coautores foráneos. Como regularidad se detecta que los firmantes más prolijos publican de forma estacional, con dos o tres documentos en el mejor año y uno o ninguno en el siguiente. Por ejemplo, de los 24 autores que produjeron al menos dos documentos en el bienio 2011-2012, 13 tienen su mayor producción en 2011, cinco la consiguen en 2012 y seis mantienen igual cantidad.

Aunque las evidencias se basan en una cantidad limitada de datos en el tiempo, existe otro argumento para sostener la hipótesis que explica el crecimiento estacional con base en los subgrupos de autores más productivos. En efecto, las investigaciones educacionales "publicables" generalmente requieren de un tiempo prolongado por la puesta en práctica del diseño experimental, cuya duración puede abarcar incluso varios meses. Esta reminiscencia neopositivista supeditada a patrones de fiabilidad y validez, todavía constituye un criterio de calidad y consecuentemente de visibilidad en las publicaciones científicas educacionales. Así van quedando excluidos los estudios mayormente cualitativos y las experiencias con pequeños grupos, aún cuando la muestra no sea fácil de incrementar.

Con el fin de clarificar la estructura general de la información, seguidamente se siguen dos caminos: un estudio horizontal que recorre por cada publicación el número de categorías afines, y otro vertical que examina el comportamiento de cada categoría en el contexto de las publicaciones educacionales cubanas de mayor visibilidad.

\subsection{Estudio horizontal: nivel de especialización de las publicaciones}

Para contabilizar las áreas del conocimiento científico educacional, respecto a las cuales cada publicación tiene relación directa, se introduce la variable SUMA $=\mathrm{C} 01+\mathrm{C} 02+\ldots+\mathrm{C} 41$, que cuenta para cada observación la cantidad de categorías generales relacionadas. Si la cantidad de categorías generales es pequeña, ello significa que las palabras clave se han concentrado en pocos estratos y recíprocamente. Por tanto, esta nueva variable describe con cierta objetividad el nivel de especialización de cada publicación. En el transcurso de la década se alcanza un promedio de 3,85 categorías por artículo (desviación típica de 1,34).

La Figura 3 ilustra los intervalos de confianza de la variable SUMA, a partir del año 2006. Las observaciones precedentes han sido suprimidas por ser relativamente escasas. De forma global se observa una tendencia lenta al decrecimiento de los valores medios por año (recta de regresión $y=-0,12 x+$ $\left.4,34, R^{2}=0,61\right)$. Las diferencias son estadísticamente significativas $(F(6,276)=2,61 ; p<0,05)$, por lo que se puede afirmar que existe una tendencia discreta hacia la especialización. La aplicación del procedimiento de contraste de diferencia mínima significativa (LSD) de Fisher produce cuatro parejas de años con diferencias significativas de sus medias: 2006-2009 (Dif. $=0,79 \pm 0,66, p$ $<0,05), 2006-2011$ (Dif. $=0,85 \pm 0,63, p<0,05$ ), 2008-2009 (Dif. $=0,65 \pm 0,50, p<0,05)$ y 20082011 (Dif. $=0,70 \pm 0,47, p<0,05$ ). La diferencia más cercana a la unidad corresponde al período 2006-2011, o sea, aparentemente se requiere de al menos un quinquenio para que se alcance un mayor nivel de especialización. 


\subsection{Estudio vertical: áreas del conocimiento}

Un estudio vertical de esta naturaleza facilita el esclarecimiento de al menos tres cuestiones importantes para la caracterización de las publicaciones, atendiendo a las categorías generales en que se ha subdividido el campo de las investigaciones educacionales. En primer lugar, la frecuencia de cada categoría refleja el interés colectivo por determinadas áreas del conocimiento científico. En segundo lugar, la búsqueda de asociaciones entre variables ayuda a clasificar el conjunto de investigaciones reportadas, aspecto que sirve de complemento para el estudio vertical, en el sentido de contraponer la especialización con los límites de la interdisciplinariedad. En tercer lugar, la determinación de factores objetivos inmanentes facilita el replanteamiento del análisis, a partir de componentes relevantes que en la multitud de variables son difíciles de distinguir.
Respecto al comportamiento específico de cada categoría, las frecuencias aparecen de forma dispar, con valores entre 1 y 95 . Después de ordenarlas de manera descendente respecto a sus frecuencias, puede observarse que las diez categorías correspondientes al primer cuartil comprenden cerca del $50 \%$ de las puntuaciones, mientras que la decena del último cuartil apenas sobrepasa el $4 \%$. En la Figura 4 se ilustran estos resultados mediante un diagrama de Pareto. Dentro del cuartil preferencial se ubican por este orden C10 (Niveles educacionales, titulaciones y organizaciones), C17 (Salud y seguridad), C11 (Procesos educacionales: perspectiva del aula), C36 (Ciencia y tecnología), C26 (Aprendizaje y percepción), C38 (Procesos y estructuras sociales), C16 (Gobierno y política), C13 (Procesos educacionales: perspectiva social), C39 (Estudiantes, profesores, personal docente), y C40 (Sujeto de instrucción).

Figura 3. Intervalos LSD de categorías afines al tesauro ERIC por año

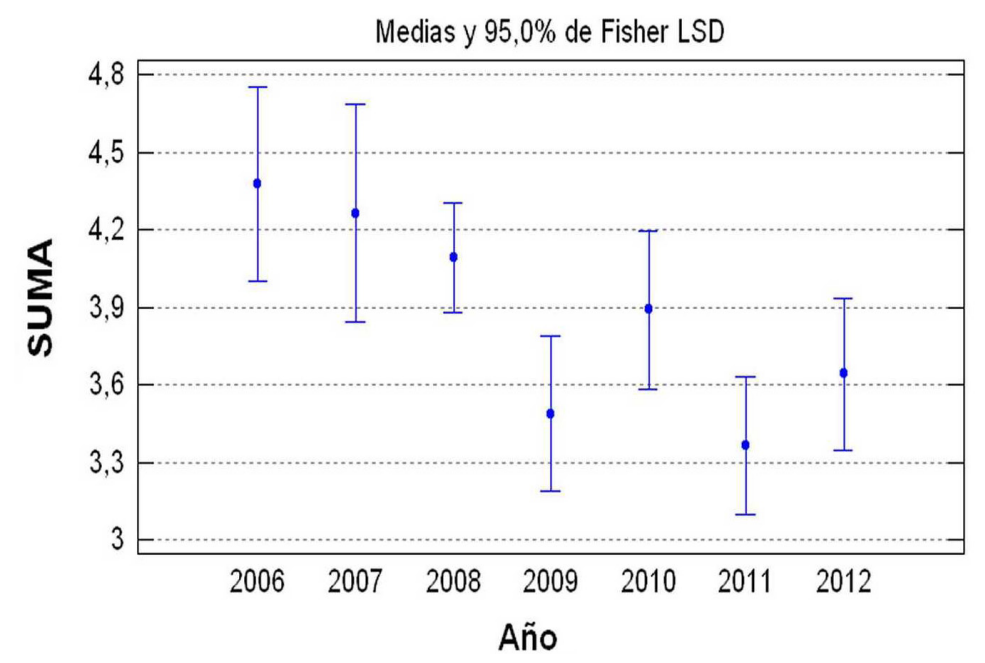

Figura 4. Diagrama de Pareto para la frecuencia absoluta de las categorías investigadas

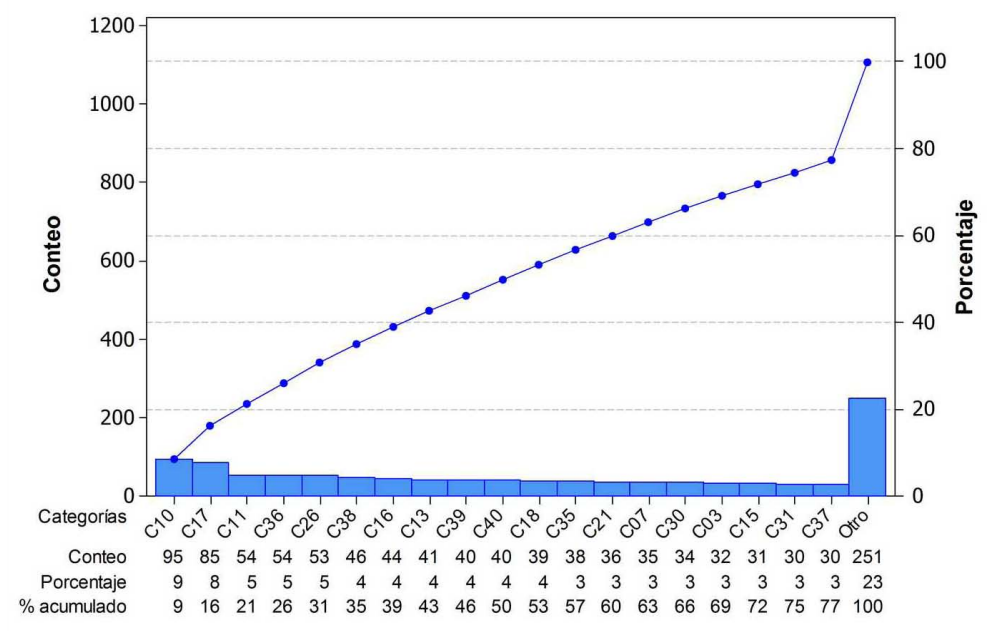


Las categorías de mayor frecuencia concuerdan plenamente con la disposición general de la matriz de datos. Por ejemplo, la primera de ellas $(\mathrm{C} 10=$ Niveles educacionales, titulaciones y organizaciones) se corresponde con publicaciones específicas de la educación superior, como la formación de profesionales y los estudios de posgrado. Seguidamente, la segunda categoría ( 17 = Salud y seguridad) refleja la numerosa cantidad de publicaciones relacionadas directa o indirectamente con el sector de la salud pública. De hecho, al conjunto de publicaciones provenientes de universidades médicas se incorporan otras realizadas en el ambiente escolar, afines a la promoción de estilos de vida sanos y responsables. Por su parte, la tercera categoría (C11 = Procesos educacionales: perspectiva del aula) da testimonio de la tendencia natural de todo docente investigador a preocuparse en primera instancia por los procesos que transcurren en el salón de clases.

El cuartil de categorías con menor frecuencia se ha integrado en la Figura 4 bajo la denominación "Otro". Allí se posicionan categorías relacionadas con las humanidades, como ocurre con la educación artística (C02 = Arte) y literaria (C34 = Lectura), con apenas una y dos observaciones respectivamente. Este hecho concuerda con hallazgos reportados por otros autores (Osca y Mateo, 2003; Bruner y Salazar, 2012; Diem y Wolter, 2013). Similarmente, en este estrecho margen también se encuentran categorías de mayor grado de especialización, como la educación física y deportiva (C32 = Educación física y recreación) y la educación especial (C08 = Discapacidad), con tres y cinco observaciones respectivamente.
La frecuencia absoluta aporta información sobre la prevalencia de una variable en el conjunto de observaciones. Esta información podría estar incompleta si se pasa por alto la posible asociación con otras variables. Por ejemplo, si las categorías C11 (Procesos educacionales: perspectiva del aula) y C26 (Aprendizaje y percepción) aparecieran regularmente en conjunción, esto significaría que los intereses investigativos se concentran en problemas de aprendizaje. Sin embargo, esto solo ocurre en 18 ocasiones, a pesar de que ambas categorías figuran entre las de mayor frecuencia. La investigación de conexiones de esta naturaleza facilita la estratificación del conjunto de publicaciones $y$, consecuentemente, su clasificación.

Con el objetivo de establecer una clasificación efectiva del conjunto de categorías, se realiza un análisis de clúster entre las variables C01, C02, ..., C41, con medida de asociación ya que se trata de variables no métricas.

La Figura 5 muestra el dendograma resultante, donde el eje vertical refleja los niveles de similitud. Puede observarse que categorías afines como C23 (Trabajo y empleo) y C30 (Ocupación) tienen un nivel de similitud superior al $80 \%$, sin embargo el problema ahora consiste en tomar una decisión acerca del nivel mínimo para el porcentaje de asociación, a fin de establecer una clasificación práctica y fiable del conjunto de variables. Esta decisión es importante si se considera que las 41 categorías generales todavía no permiten distinguir con facilidad las tendencias en las investigaciones educacionales cubanas, a partir de las publicaciones de mayor visibilidad.

Figura 5. Dendograma para el estudio de conglomerados de categorías

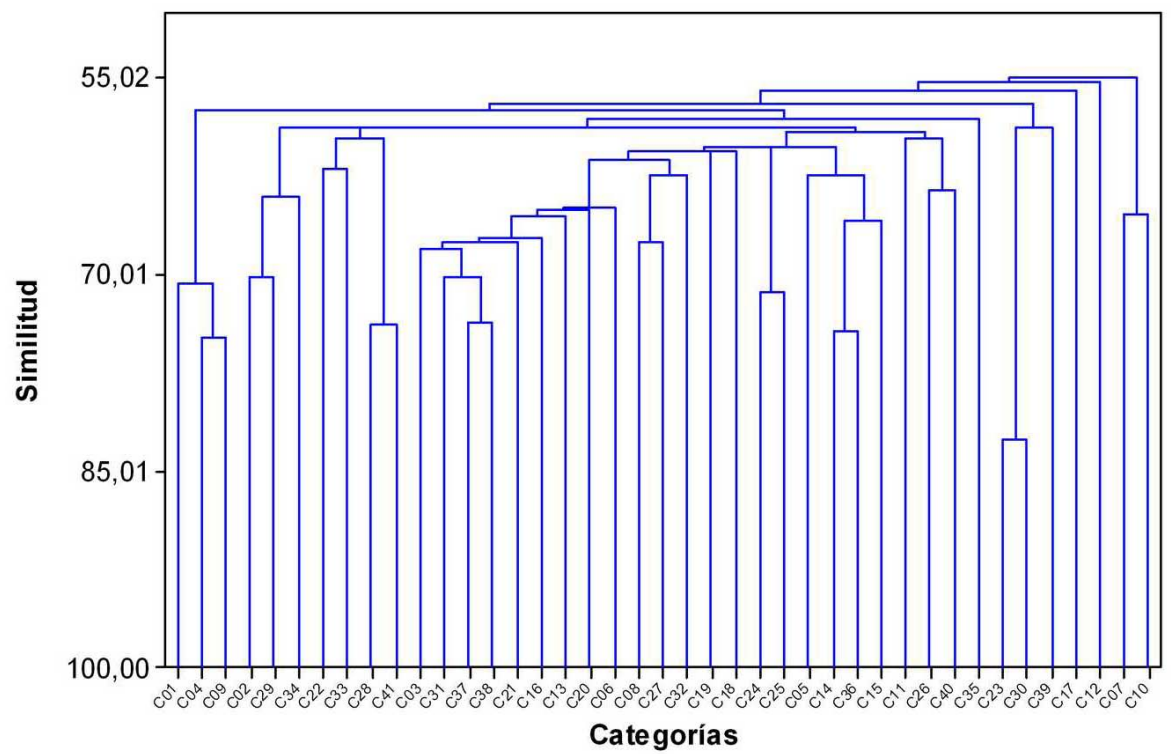


Ya que la mayoría de las divisiones tienen lugar cerca del $60 \%$ de similitud, se toma este valor como criterio para establecer la clasificación de las variables. Para este nivel se tiene un conjunto de siete conglomerados, los cuales se describen a continuación.

1. Conglomerado I (variable $\mathrm{C} 12=$ Procesos educacionales: perspectiva de la escuela), que refiere investigaciones relacionadas con la escuela como institución, donde tienen lugar procesos complejos y dinámicos. Aunque la frecuencia absoluta de esta variable es baja, se encuentra bien localizada.

2. Conglomerado II (variable $\mathrm{C} 17$ = Salud y seguridad), que apunta hacia los campos de la educación para la salud y de la formación de profesionales de la salud.

3. Conglomerado III (variable C35 = Investigación y teoría), donde el tema central lo ocupan aspectos teóricos tales como la modelación de procesos, el establecimiento de estrategias, la fundamentación de principios, entre otros.

4. Conglomerado IV (variables C07 = Organización del currículo y $\mathrm{C} 10$ = Niveles educacionales, titulaciones y organizaciones), que pone de manifiesto un interés creciente por perfeccionar los currículos universitarios y de diferentes programas de formación posgraduada.

5. Conglomerado V (variables $\mathrm{C} 23=$ Trabajo $\mathrm{y}$ empleo, C30 = Ocupación y C39 = Estudiantes, profesores, personal docente), relacionado con el perfeccionamiento de la labor de los profesionales de la educación por medio del denominado "trabajo metodológico" (Art. 24, Resolución 2010/07 del MES), así como una preocupación por acercar a los estudiantes universitarios al contexto de su futura profesión.

6. Conglomerado VI (variables $\mathrm{C} 01=$ Agricultura y fuentes naturales, C04 = Empresa, comercio e industria y $\mathrm{C09}$ = Economía y finanzas), que integra aspectos relacionados con la educación económica y medioambiental.

7. Conglomerado VII (variables restantes), donde se agrupan aquellos campos de investigación poco frecuentes pero correlacionados, como C28 (Medida) y C41 (Test y escalas) que aparecen principalmente en estudios diagnósticos; y también algunos campos de frecuencia superior a la media, pero menos correlacionados como C11 (Procesos educacionales: perspectiva del aula), C26 (Aprendizaje y percepción) y C36 (Ciencia y tecnología), con énfasis en las tecnologías de la información y las comunicaciones.

Para culminar el estudio vertical se realiza un análisis factorial respecto a las variables (tipo R), con la finalidad de identificar factores subyacentes que potencialmente sirvan para explicar posibles relaciones de interdependencia, dentro de una va- riabilidad grande de los datos. La medida de adecuación de la muestra (MSA por sus siglas en inglés) produce el valor $M S A=0,55$. Esto sugiere emplear la técnica con ciertas reservas, pues para MSA $<0,5$ ya se considera inaceptable de acuerdo con la clasificación propuesta por Kaiser y Rice (1974). El test de esfericidad de Bartlett, sin embargo, rechaza la hipótesis de diagonalidad de la matriz de correlación indicando que sí existen relaciones significativas entre las variables $\left(\chi^{2}(820)\right.$ $=2118,19 ; p<0,01)$. Resultan 16 componentes, lo cual reduce las variables a menos de la mitad.

La extracción solo produce cuatro variables iniciales con comunalidades menores que 0,50 , lo cual es favorable. Sin embargo, los 16 componentes extraídos apenas explican el $63,44 \%$ de la varianza total y los dos primeros el $11,25 \%$, después de efectuar la rotación ortogonal de factores por el método Equamax. En el primer componente las mayores puntuaciones factoriales se concentran en las variables C03 (Prevención y equidad), C13 (Procesos educacionales: perspectiva social), C16 (Gobierno y política), C21 (El individuo en su contexto social), C31 (Personas y culturas), C37 (Problemas sociales) y C38 (Procesos y estructuras sociales), por lo que puede decirse que se trata de un factor de naturaleza sociocultural de las investigaciones educacionales publicadas. En el segundo componente, las mayores puntuaciones factoriales están en C14 (Equipamientos), C15 (Instalaciones) y C36 (Ciencia y tecnología), lo cual sugiere una especie de factor tecnológico-instrumental, por referir principalmente estudios de laboratorio, uso de paquetes computacionales, acceso a Internet, entre otros aspectos.

En la Figura 6 aparece la gráfica de cargas para los dos primeros factores. Los ejes de coordenadas permiten observar una distribución equitativa de las cargas en los cuatro cuadrantes $(11,10,10$ y 10 en los cuadrantes I-IV respectivamente). De aquí se desprende que al menos uno de los dos factores se manifiesta positivamente en cerca de las tres cuartas partes de las variables estudiadas. Tras una simple inspección también puede comprobarse la relativa cercanía de los conglomerados I, II, IV y VI al origen de coordenadas, lo cual significa que las publicaciones allí incluidas corresponden a investigaciones con tendencia neutral respecto a los factores anteriormente descritos. Un aspecto realmente significativo, pues constituyen estratos de investigaciones educacionales donde aspectos tan relevantes como lo tecnológico y lo social no son tomados en consideración de forma primordial.

En el gráfico las variables C11, C12 y C13 aparecen circuladas. Como ya se había señalado, se trata de categorías que describen los procesos educacionales desde perspectivas micro (el salón de clases), meso (la escuela) y macro (la sociedad). La cercanía común de las ordenadas de estas tres variables al valor nulo sugiere que los estudios centrados en los procesos educacionales están re- 
Figura 6. Gráfica de carga respecto a dos componentes como factores subyacentes

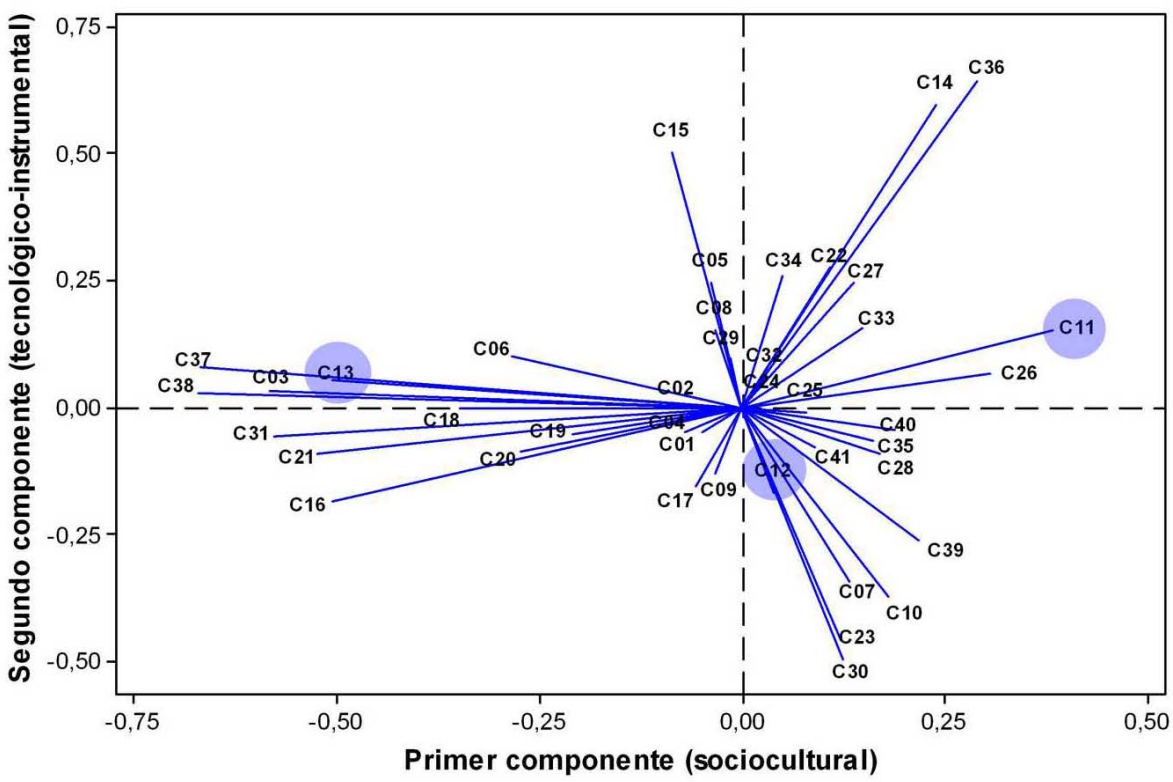

lativamente separados de las investigaciones teóricas y tecnológicas. Nótese, por ejemplo, que el relativo aislamiento de $\mathrm{C} 14$ (Equipamientos) y C36 (Ciencia y tecnología) parece indicar que los estudios de este tipo, además de tender a ser un fin en sí mismos, están muy asociados al contexto sociocultural. Una manifestación de ello consiste en la sustitución paulatina de las siglas NTIC (Nuevas Tecnologías de la Información y las Comunicaciones), sencillamente por TIC, ya que constituyen un fenómeno característico de la moderna sociedad del conocimiento donde la otrora novedad se sustituye en lo sucesivo por la cotidianeidad.

Desde una lógica apriorística podría suponerse que la disposición más a la izquierda de C13 (Procesos educacionales: perspectiva social) no concuerda con la concepción hipotética del factor sociocultural. En efecto, era de esperar que las investigaciones afines a los procesos educacionales, desde una perspectiva social, aparecieran dispuestas en el sentido positivo del primer componente. Sin embargo, las evidencias reflejan que esto no es así. Todo parece indicar que existe una tendencia a investigar la perspectiva social como un proceso predominantemente de adentro hacia afuera. Por ejemplo, cuando se estudia la calidad educacional ("Educational Quality" en el tesauro ERIC) predomina el interés por la calidad del encargo social, como es el caso de la formación de un profesional competente, con base en objetivos curriculares preestablecidos. En cambio, solo esporádicamente se tiene en cuenta la percepción externa sobre la calidad del encargo social, el grado de satisfacción de la formación recibida, el posicionamiento insti- tucional, entre otros aspectos que constituyen una mirada de afuera hacia adentro.

\section{CONCLUSIONES}

El objetivo central del presente estudio ha consistido en caracterizar las publicaciones científicas cubanas del contexto educacional, desde el prisma de las bases de datos WoS y Scopus. Toda caracterización se diferencia de una descripción por procurar mayor precisión en la distinción de los rasgos fundamentales de un objeto o fenómeno y también por un mayor grado de generalización. Por tanto, se impone ahora una síntesis de los aspectos más relevantes acontecidos durante la investigación, así como un análisis crítico de los mismos.

De manera general, las publicaciones sometidas a escrutinio se caracterizan por lo siguiente:

1. Producción científica discreta con tendencia a un rápido crecimiento, con altibajos probablemente ocasionados por la influencia de las publicaciones de un subgrupo de autores más prolijos.

2. Índices de citaciones y de internacionalización relativamente bajos, con predominio del trabajo colectivo sobre el individual.

3. Preponderancia de publicaciones en revistas relacionadas con el campo de la salud, lo cual no parece ser un fenómeno local sino una regularidad internacional.

4. Tendencia a la especialización dentro de los objetos de investigación. 
5. Existencia de una tipología que sugiere clasificar los estudios en siete grupos fundamentales.

6. Presencia de dos ejes transversales que facilitan una mirada crítica al conjunto de investigaciones reportadas: los componentes sociocultural y tecnológico-instrumental.

Es importante puntualizar que estas características no se refieren a la producción científica educacional cubana, sino a la de mayor visibilidad por medio de una muestra que ha procurado ser representativa. Desafortunadamente, los autores no han encontrado un estudio bibliométrico similar sobre la producción científica nacional en el campo de la educación, a fin de efectuar un análisis comparado. La revisión muy superficial (apenas de los títulos) de más de 500 tesis doctorales de todo el país, almacenadas en el repositorio de la Universidad de Ciencias Pedagógicas "José de la Luz y Caballero", hace suponer que existen diferencias cardinales, pero esto ya es terreno fértil para un estudio ulterior.

La presente investigación pone de manifiesto una vez más la importancia de la normalización de términos científicos por medio de los tesauros. Particularmente, la existencia de niveles de clasificación ha permitido efectuar el estudio con base en las categorías generales del tesauro ERIC. Esto es positivo en el sentido de la simplificación del número de variables y la consecuente facilitación del procesamiento estadístico. Sin embargo, el conjunto de categorías presenta un nivel de generalidad tal que el componente pedagógico queda relativamente marginado. Una tentativa por lograr mayor objetividad en el análisis podría tomar en cuenta las siguientes posibilidades:

1. Jerarquizar el conjunto de palabras clave, a fin de esclarecer la naturaleza de la investigación con un número más reducido de ellas.

2. Redefinir las variables a partir de otros aspectos esenciales de las investigaciones educacionales (rama de la ciencia, nivel de enseñanza, tipo de investigación, tipo de resultado científico, etcétera).

La simplificación del número de variables puede allanar el camino para caracterizar mejor las investigaciones reportadas. En ocasiones existen cuestiones de interés para la cienciometría que no se contemplan durante la enumeración de palabras clave. Por ejemplo, si se trata de una investigación a ciclo completo $(\mathrm{I}+\mathrm{D}+\mathrm{i})$, el tipo de conocimiento que genera (básico o aplicado), el grado de generalización de los resultados, el tipo de proyecto financiado, entre otros aspectos.

A pesar del esfuerzo por obrar con parsimonia y objetividad, la práctica siempre impone restricciones que es justo señalar. La cantidad de observaciones no es lo suficientemente grande como se quisiera, de modo que las conjeturas realizadas sobre el fenómeno de las estacionalidades y los re- sultados del análisis factorial deben manejase con cautela. Asimismo, la estructura subyacente basada en sendos componentes constituye una síntesis que puede percibirse de muchas maneras, en dependencia de las cargas factoriales y de la valoración de cada investigador. A pesar de que la varianza explicada por los dos primeros componentes es baja, su estudio no deja de ser oportuno por constituir dos aspectos relevantes para el contexto educacional contemporáneo. Incluso todavía el análisis puede enriquecerse con la incorporación de una tercera variable latente, aunque ello dilataría sensiblemente la discusión de los resultados.

Tampoco puede evitarse cierta subjetividad en el proceso de reclasificación de categorías generales para cada artículo, donde lo ideal sería la lectura primaria del contenido de cada documento en lugar de las palabras clave y del resumen. Si bien este ideal deja de ser práctico cuando el tamaño de las observaciones crece, incluso el procesamiento de las palabras claves y de los resúmenes se complican también cuando no existe normalización.

De forma general, una concepción holística de las ciencias de la educación complejiza el empeño por conseguir un estudio de tipo censal-documental. Para lograr mayor proximidad a la población se requiere de la mejora continua de los métodos de búsqueda, y para obtener mejores frutos en el análisis de contenido se precisa de nuevos progresos en los métodos de procesamiento.

A tenor de los hallazgos anteriores, conviene profundizar más en la identificación de indicadores cienciométricos que caractericen mejor la producción científica educacional, a partir de su visibilidad. Varios aspectos importantes se han tomado en consideración y suscitan numerosas implicaciones, pero queda todavía pendiente la determinación de su esencialidad. Los límites de una ciencia relativamente joven como la cienciometría conducen a solapar conceptos cienciométricos con bibliométricos y con ello a confundir desarrollo científico con visibilidad. Sin duda son obstáculos que cada estudio ulterior irá franqueando paulatinamente.

\section{AGRADECIMIENTOS}

Esta investigación ha recibido apoyo del proyecto AECID A2/039476/11: Fortalecimiento Institucional en la Enseñanza de la Estadística, la Investigación Operativa, Fiabilidad, y Calidad y Aplicación Científica en Temas de Interés Regional.

\section{BIBLIOGRAFÍA}

Agarwal, P. K. (2012). Advances in cognitive psychology relevant to education: Introduction to the special issue. Educational Psychology Review, vol. 24 (3), 353-354. http://dx.doi. org/10.1007/s10648-012-9212-0

Anta, C. (2008). Análisis bibliométrico de la investigación educativa divulgada en publicaciones 
periódicas españolas entre 1990-2002. Revista Electrónica de Investigación Educativa, vol. 10 (1). Disponible en: http://redie.uabc.mx/index. php/redie/article/download/185/323 [Recuperado: 10/08/2013].

Araujo, J. A.; Van Hooydonk, G.; Torricella, R. G.; Arencibia, R. (2005). Cuban scientific articles in ISI Citation Indexes and CubaCiencias databases (1988-2003). Scientometrics, vol. 65 (2), 161-171. http://dx.doi.org/10.1007/s11192005-0265-4

Archambault, E.; Larivière, V. (2010). The limits of bibliometrics for the analysis of the social sciences and humanities literature. En: World Social Sciences Report (pp. 251-254). International Social Science Council: UNESCO Publishing. Disponible en: http://unesdoc.unesco.org/ images/0018/001883/188333e.pdf [Recuperado: 03/12/2011].

Arencibia, R.; de Moya, F. (2010). Challenges in the study of Cuban scientific output. Scientometrics, vol. 83 (3), 723-737. http://dx.doi. org/10.1007/s11192-009-0150-7

Brunner, J. J.; Salazar, F. A. (2012). Investigación educacional en Iberoamérica: entre la invisibilidad y la medición. Magis, Revista Internacional de Investigación en Educación, vol. 4 (9), 559-575.

Carpallo, A.; Burgos, E. (2008). Estudio bibliométrico y de calidad de la Revista Complutense de Educación (1990-2007). Revista Complutense de Educación, vol. 19 (1), 13-29.

Chang, Y. H.; Chang, Ch. Y. Tseng, Y. H. (2010). Trends of science education research: an automatic content analysis. Journal of Science Education and Technology, vol. 19, 315-331. Disponible en: http://140.122.100.145/topntnu/ top/data/2_2_1.pdf [Recuperado: 10/08/2013]. http://dx.doi.org/10.1007/s10956-009-9202-2

Diem, A.; Wolter, S. C. (2013). The use of bibliometrics to measure research performance in education sciences. Research in Higher Education, vol. 54 (1), 86-114. http://dx.doi.org/10.1007/ s11162-012-9264-5

Fernández, A.; Torralbo, M.; Rico, L.; Gutiérrez, P.; Maz, A. (2003). Análisis cienciométrico de las tesis doctorales españolas en Educación Matemática (1976-1998). Revista Española de Documentación Científica, vol. 26 (2), 162-176.

Gantman, E. R. (2011). La productividad científica argentina en Ciencias Sociales: Economía, Psicología, Sociología y Ciencia. Política en el CONICET (2004-2008). Revista Española de Documentación Científica, vol. 34 (3), 408-425. http://dx.doi.org/10.3989/redc.2011.3.829

Göktaş, Y. y otros (2012). Trends in Educational Research in Turkey: A Content Analysis. Educational Sciences: Theory \& Practice, vol. 12 (1), 455-459. Disponible en: http://files.eric. ed.gov/fulltext/EJ978453.pdf [Recuperado: 10/08/2013].
González, J. P.; Reyes, R. (2010). Desarrollo de la educación en Cuba después del año 1959. Revista Complutense de Educación, vol. 21 (1), 13-35.

Gonçalves, A. J. (2010). Educação inclusiva em revistas pedagógicas: análises de conteúdo e bibliométrica. Revista Brasileira de Educação Especial, vol. 16 (3), 479-494. Disponible en: http://www.scielo.br/pdf/rbee/v16n3/ v16n3a11.pdf [Recuperado: 10/08/2013].

Hemphill, M. A.; Richards, K. A. R.; Templin, T. J.; Blankenship, B. T. (2012). A content analysis of qualitative research in the Journal of Teaching in Physical Education from 1998 to 2008. Journal of Teaching in Physical Education, vol. 31, 279287. Disponible en: http://www.naspspa.org/ AcuCustom/Sitename/Documents/DocumentItem/06_hemphill_JTPE_20110075_279-287.pdf [Recuperado: 10/08/2013].

Hicks, D. (1999). The difficulty of achieving full coverage of international social science literature and the bibliometric consequences. Scientometrics, vol. 44 (2), 193-295. http://dx.doi. org/10.1007/BF02457380

Kaiser, H. F.; Rice, J. (1974). Little jiffy, mark IV. Educational and Psychological Measurement, vol. 34 (1), 111-117. http://dx.doi. org/10.1177/001316447403400115

Lee, M. H.; Wu, Y. T.; Tsai, Ch. Ch. (2009). Research trends in science education from 2003 to 2007: A content analysis of publications in selected journals. International Journal of Science Education, vol. 31 (15), 1999-2020. http:// dx.doi.org/10.1080/09500690802314876

López, P. (1996). Introducción a la Bibliometría. Valencia: Promolibro.

Medina, J. M.; Fernández, I. M.; Gil, J. A.; Fernández, A. (2008). La investigación odontológica española en la base Science Citation Index: un estudio cienciométrico (1974-2006). Revista Española de Documentación Científica, vol. 31 (2), 169-189.

Mochón, G.; Sorli, Á. (2007).Tesauros de ciencias sociales en Internet. Revista Española de Documentación Científica, vol. 30 (3), 395-419.

Moed, H. F. (2005). Citation Analysis in Research Evaluation. Dordrecht (The Netherlands): Springer.

Novo, M.; Bautista, M. J. (2012). Análisis de la incidencia de la educación ambiental para el desarrollo sostenible en las revistas científicas españolas. Revista de Educación, vol. 358, 583-597.

Osca, J.; Mateo, M. E. (2003). Difusión de las revistas españolas de ciencias sociales y humanidades. Acercamiento bibliométrico. Revista General de Información y Documentación, vol. 13 (1), 115-132.

Pérez, A. M.; López, M. P.; Poveda, P. (2009). Aprendizaje cooperativo y formación del profesor. Un estudio bibliométrico (1997-2008). Anales de Documentación, vol. 12, 209-220. Disponible en: http://digitum.um.es/xmlui/ 
bitstream/10201/9687/1/p.\%20209-220.pdf [Recuperado: 10/08/2013].

Reglamento para el trabajo docente y metodológico en la educación superior. Resolución 210, 2007, Ministerio de Educación Superior. Gaceta Oficial de la República de Cuba, No. 040 (pp. 209-232), La Habana. Disponible en: http:// www.gacetaoficial.cu/pdf/GO X_040_2007.rar [Recuperado: 27/08/2013].

SCImago Research Group (2007). SJR - SCImago Journal \& Country Rank. Disponible en: http:// www.scimagojr.com [Recuperado: 17/07/2013].

Torres, P. (2012). La visibilidad internacional de los resultados de la investigación educativa: ¿Cómo estamos los cubanos? Revista Transformación, vol. 8 (2), 114-128. Disponible en: http://www.ucp.cm.rimed. cu/uzine/transformacion/2012/2012.12.02/articulos/12_torres.fernandez.paul-visibilidad.internacional.pdf [Recuperado: 12/07/2013].
Vallejo, M.; Ocaña, A.; Bueno, Á.; Torralbo, M.; Fernández, A. (2005). Producción científica sobre educación multicultural contenida en las bases de datos Social Science Citation Index y Arts \& Humanities Citation Index (1956-2003). Revista Española de Documentación Científica, vol. 28 (2), 206-220.

Van Leeuwen, T. (2006). The application of bibliometric analyses in the evaluation of social science research. Who benefits from it, and why it is still feasible. Scientometrics, vol. 66 (1), 133-154. http://dx.doi.org/10.1007/s11192-006-0010-7

Vinluan, L. R. (2012). Research productivity in education and psychology in the Philippines and comparison with ASEAN countries. Scientometrics, vol. 91 (1), 277-294. http://dx.doi. org/10.1007/s11192-011-0496-5 